\title{
Assessment of Serum Tumor Markers for Predicting Ocular Metastasis in Lung Adenocarcinoma: A Retrospective Study
}

\author{
Wen-Qing Shi, ${ }^{1}$ Wen-Feng Liu $\mathbb{D}^{2}{ }^{2}$ Biao Li, ${ }^{1}$ Qi Lin, ${ }^{1}$ Qing-Hai Li, ${ }^{1}$ Yu-Qing Zhang, \\ Qing Yuan, ${ }^{1}$ Rong-Bin Liang, ${ }^{1}$ Qian-Min Ge, ${ }^{1}$ and Yi Shao $\mathbb{D}^{1}$ \\ ${ }^{1}$ Department of Ophthalmology, The First Affiliated Hospital of Nanchang University, Jiangxi Province Clinical \\ Ophthalmology Institute, Nanchang, 330006 Jiangxi, China \\ ${ }^{2}$ Department of Hepatic Oncology, Zhongshan Hospital, Fudan University, Shanghai 200032, China
}

Correspondence should be addressed to Yi Shao; freebee99@163.com

Received 5 October 2019; Revised 1 February 2020; Accepted 4 June 2020; Published 25 June 2020

Academic Editor: Wen-Jun Tu

Copyright ( 2020 Wen-Qing Shi et al. This is an open access article distributed under the Creative Commons Attribution License, which permits unrestricted use, distribution, and reproduction in any medium, provided the original work is properly cited.

\begin{abstract}
The purpose of this study was to detect clinical variations between lung adenocarcinoma patients with and without ocular metastasis $(\mathrm{OM})$ to identify risk factors for $\mathrm{OM}$ and assess the diagnostic values. We included 1153 patients with lung adenocarcinoma in this study. Independent $t$-tests and chi-square tests were used to compare patients' clinical characteristics. Statistically significant parameters were analyzed by binary logistic regression to detect risk factors of OM. The results showed that the OM group had increased alpha-fetoprotein (AFP), carcinoembryonic antigen (CEA), cytokeratin fragment 19 (CYFRA 21-1), carbohydrate antigen- (CA-) 125, CA-153, and total prostate-specific antigen (TPSA) compared with the NOM group. CYFRA21-1 is the most useful biomarker for detecting OM in this population.
\end{abstract}

\section{Introduction}

Lung cancer is a common malignant tumor worldwide. The incidence and mortality of lung cancer rank first among all cancer types in males, accounting for $17 \%$ of all new cancer cases and $23 \%$ of cancer-related deaths [1]. The female incidence of lung cancer has continually increased in recent years, and it is now the second most common type of cancer in women [2]. Causes of lung cancer include air pollution and exposure to occupational and environmental carcinogens [3, 4]. Normal epithelial cells experience multiple genetic insults and eventually undergo abnormal growth and develop invasive behaviors. Lung cancer can be divided into small cell and nonsmall cell according to the histological type. The latter group includes squamous cell cancer, adenocarcinoma, and large cell cancer. Most lung adenocarcinomas originate from the bronchial mucosal epithelium, while a few develop from the mucous glands of bronchia. However, the prognosis of lung adenocarcinoma is poor $[5,6]$.

The eye is a rare metastatic site for malignant tumors. Ocular metastasis $(\mathrm{OM})$ is the most often secondary to, for example, breast, lung, kidney, or prostate cancer $[7,8]$. Lung adenocarcinoma is usually diagnosed at an advanced stage, and patients often have distant metastases [9]. The most common sites are the brain, bone, liver, and adrenal gland $[10,11]$. In the early stage, OM may have not been accompanied by ophthalmic symptoms. Over time, patients with OM may have blurred vision, pain, visual field defects, flashes, and diplopia [12]. OM can also result in pupil deformation and secondary glaucoma [13]. These symptoms seriously affect the quality of life and shorten the survival time of patients with lung adenocarcinoma. Some studies have also reported asymptomatic cases of choroidal metastasis [14, 15]. Given the shortcomings of conventional computed tomography (CT) and magnetic resonance imaging (MRI) in detecting eye metastases, a simple and economical technique for predicting eye metastases would be clinically useful.

Tumor markers are widely used for clinical screening. Detection of markers in the blood, body fluid, or cells can facilitate diagnosis, help clarify the pathogenesis, and inform prognosis. Many serum tumor markers are associated with lung cancer. For example, Ma et al. [16] found increased 
levels of carcinoembryonic antigen (CEA), carbohydrate antigen- (CA-) 125, and cytokeratin fragment 19 (CYFRA21-1) in lung cancer patients, which could be used for diagnostic purposes. Previous studies have also shown that CEA, CYFRA21-1, and CA-125 are associated with worse prognosis in non-small-cell lung cancer [17].

Tumor markers may be effective markers for predicting metastasis. However, it is not clear whether there are differences in tumor markers used in the diagnosis of $\mathrm{OM}$ and NOM patients with lung adenocarcinoma. In this study, we collected medical records of lung adenocarcinoma patients treated at the First Affiliated Hospital of Nanchang University and retrospectively analyzed the diagnostic value of several tumor markers, which might provide a medical basis for predicting $\mathrm{OM}$ in this population.

\section{Materials and Methods}

2.1. Study Design. All patients volunteered to participate. This study was approved by the Medical Research Ethics Committee of the First Affiliated Hospital of Nanchang University. The patients were diagnosed with lung adenocarcinoma between October 2001 and February 2017 based on pathological sections obtained by surgical excision or biopsy. $\mathrm{OM}$ was diagnosed by CT and MRI. Patients with primary ocular malignancies, benign ocular tumors, and secondary lung cancer were excluded.

2.2. Data Collection. We collected clinical data including age, sex, and treatment from the patients' medical records. Some tumor markers were also detected, including calcium, hemoglobin (HB), alkaline phosphatase (ALP), alpha-fetoprotein (AFP), CEA, neuron-specific enolase (NSE), CYFRA 21-1, CA-125, CA-153, CA-199, and total prostate-specific antigen (TPSA). All data were collected when the patients were diagnosed with lung adenocarcinoma.

2.3. Statistical Analysis. We performed independent $t$-tests (age, tumor markers) and chi-square tests (sex) to compare the OM and NOM groups. Binary logistic regression models were then applied to identify independent risk factors for $\mathrm{OM}$. We then constructed receiver operating characteristic (ROC) curves and areas under the curves (AUCs). Excel 2010 software (Microsoft, Washington, USA) was used to calculate the cutoff value, sensitivity, and specificity of each biomarker. Differences were considered significant at $p<$ 0.05. All statistical analyses were performed using SPSS 20.0 software (SPSS, IBM, USA) and Excel 2010 software.

\section{Results}

3.1. Demographics and Clinical Characteristics. We recruited 1153 patients (47 OM, $1106 \mathrm{NOM}$ ). The mean ages of the $\mathrm{OM}$ and NOM groups were $59.0 \pm 1.5$ and $59.2 \pm 0.3$ years, respectively. No significant differences were noted in sex or age between the OM and NOM groups $(p>0.05)$. Full details are shown in Table 1 and Figure 1. Other metastatic sites of the OM group were the lung (38.3\%), lymph node (59.6\%), brain $(97.9 \%)$, bone $(57.4 \%)$, liver $(17.0 \%)$, peritoneum $(4.3 \%)$, and pleura (6.4\%), while metastatic sites of the
TABle 1: The clinical characteristics of patients with lung adenocarcinoma.

\begin{tabular}{lccc}
\hline Patient characteristics & $\begin{array}{c}\text { OM group } \\
(n=47)\end{array}$ & $\begin{array}{c}\text { NOM group } \\
(n=1106)\end{array}$ & $p$ value \\
\hline Gender $^{\mathrm{a}}$ & 33 & 657 & 0.139 \\
$\quad$ Male & 14 & 449 & \\
Female & & & \\
Age $^{\mathrm{b}}$ & $59.0 \pm 1.5$ & $59.2 \pm 0.3$ & 0.927 \\
$\quad$ Mean & & & \\
Treatment & 6 & 286 & \\
$\quad$ Surgery & 42 & 660 & \\
$\quad$ Chemotherapy & 12 & 74 & \\
$\quad$ Radiotherapy & 4 & 169 & \\
Symptomatic treatment & 4 & & \\
\hline
\end{tabular}

${ }^{\mathrm{a}} \mathrm{A}$ chi-square test was applied. ${ }^{\mathrm{b}}$ Student's $t$-test was applied. $p<0.05$ was thought to be statistical significance. OM: ocular metastasis; NOM: nonocular metastasis.

NOM group were the lung (32.5\%), lymph node (61.7\%), brain $(16.0 \%)$, bone $(35.2 \%)$, liver $(15.8 \%)$, peritoneum $(12.4 \%)$, and pleura (0.5\%). See more details in Table 2.

3.2. Differences in the Clinical Features and Risk Factors of Patients with OM. There were no significant differences in the levels of calcium, HB, ALP, NSE, or CA-199 between the OM and NOM groups $(p>0.05)$. However, we found higher levels of AFP, CEA, CYFRA 21-1, CA-125, CA-153, and TPSA in the OM group $(p<0.05)$. Detailed results are listed in Table 3. Binary logistic regression modeling identified CYFRA 21-1, CA-125, and CA-153 as independent risk factors of OM in patients with lung adenocarcinoma. Details are shown in Table 4.

3.3. Cutoff Values, AUCs, Sensitivities, and Specificities of Biomarkers for OM Diagnosis. ROC curves (Figure 2) showed that the AUCs for CYFRA 21-1, CA-125, and CA-153 were $0.928,0.749$, and 0.758 , respectively. The cutoff values of CYFRA 21-1, CA-125, and CA-153 were $6.785 \mathrm{ng} / \mathrm{mL}$, $66.295 \mathrm{U} / \mathrm{mL}$, and $13.005 \mathrm{U} / \mathrm{mL}$, respectively. The sensitivities and specificities of diagnosing OM by CYFRA21-1 were $93.6 \%$ and $79.4 \%$, by CA- 125 , they were $74.5 \%$ and $74.4 \%$, and by CA- 153 , they were $83.0 \%$ and $58.0 \%$, respectively (Table 5). Combinations of the three factors were also calculated, and Figure 3 shows the ROC curves for CYFRA 21-1 +CA-125, CYFRA 21-1+CA-153, CA-125+CA-153, and CYFRA 21-1+CA-125+CA-153. The highest AUC value was for CYFRA 21-1.

\section{Discussion}

Lung adenocarcinoma has become one of the most common diseases with high morbidity and mortality. $\mathrm{OM}$ is rare in lung adenocarcinoma patients, but the clinical symptoms are serious. OM seriously affects the quality of life of patients and may suggest poor prognosis, so early prediction is of great significance. 

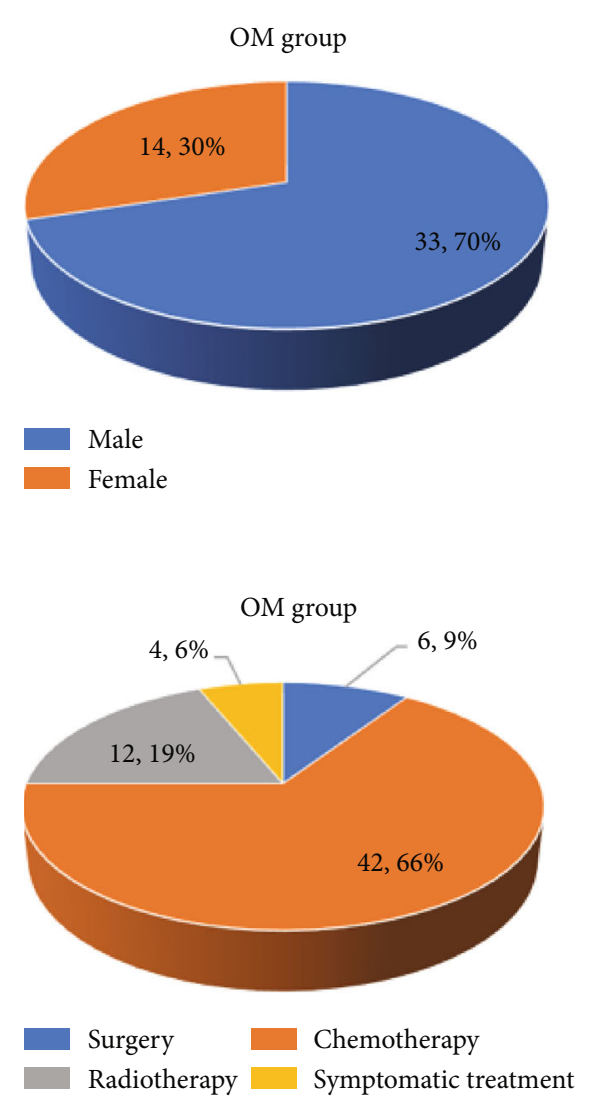

Gender

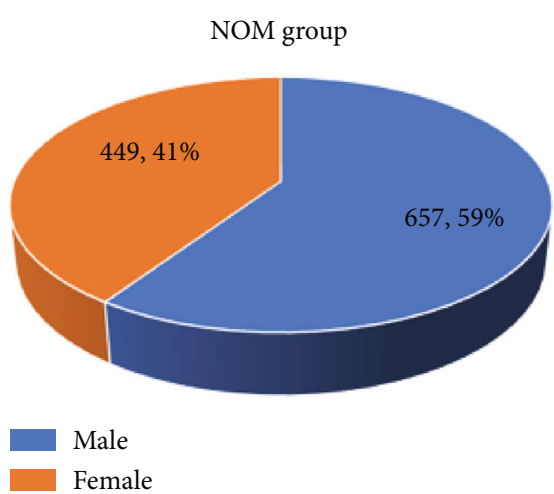

(a)

Treatment

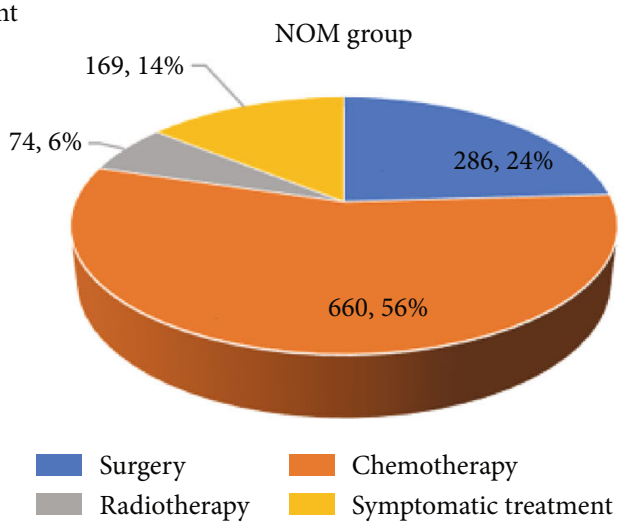

(b)

FIGURE 1: The clinical characteristics of patients with lung adenocarcinoma. OM: ocular metastasis; NOM: nonocular metastasis.

TABLE 2: Other metastatic sites of the OM and NOM groups.

\begin{tabular}{lcc}
\hline Sites & OM & NOM \\
\hline Lung & $18(38.3 \%)$ & $359(32.5 \%)$ \\
Lymph node & $28(59.6 \%)$ & $682(61.7 \%)$ \\
Brain & $46(97.9 \%)$ & $177(16.0 \%)$ \\
Bone & $27(57.4 \%)$ & $389(35.2 \%)$ \\
Liver & $8(17.0 \%)$ & $175(15.8 \%)$ \\
Peritoneum & $2(4.3 \%)$ & $137(12.4 \%)$ \\
Pleura & $3(6.4 \%)$ & $6(0.5 \%)$ \\
\hline
\end{tabular}

OM: ocular metastasis; NOM: nonocular metastasis.

OM that appears in lung cancer patients is caused by hematogenous dissemination of tumor cells [18]. In a study including 229 eyes of 194 patients with uveal metastasis [19], tumors were located in the choroid (88\%), iris (10\%), and ciliary body (2\%), with bilateral uveal (18\%). The choroid is supplied by several thick, short posterior ciliary arteries in the rear of the eye, and there are extensive anastomotic branches between the choroidal vessels. The lumen is larger, and blood flow is abundant but slow [20]. For these reasons, choroidal metastasis is the most common clinical presentation. Metastasis to the orbit, eyelid, conjunctiva, retina, and optic nerve has also been reported [21, 22]. Interestingly,
$\mathrm{OM}$ is more common in the left eye. This is because the left common carotid artery branches directly from the aortic arch, allowing tumor emboli to easily enter the left eye, whereas right eye access requires bypassing the anonymous artery.

Traditional histopathological and immunohistochemical examinations and cytogenetic analyses are tedious and time-consuming. Once a diagnosis of lung cancer is made, physicians can choose multimodal treatment including surgery, radiotherapy, and chemotherapy. Tumor markers mainly exist in serum and can be detected by immunological, biological, and chemical methods [23]. Compared with CT and MRI, they are economical and convenient. Most importantly, tumor markers have a predictive value. Table 6 shows the risk factors for distant metastasis of lung cancer reported in previous studies.

After analyzing clinical data from 1153 patients with lung adenocarcinoma, we found that the concentrations of AFP, CEA, CYFRA 21-1, CA-125, CA-153, and TPSA were significantly elevated in patients with OM. However, AFP is associated with liver metastasis [24], and TPSA is associated with benign prostatic hyperplasia, prostate cancer, and bone metastasis $[25,26]$. We therefore excluded AFP and TPSA from the tumor markers under consideration as OM markers. According to the binary logistic regression result, 
TABLE 3: Differences of tumor markers between lung adenocarcinoma patients with and without OM.

\begin{tabular}{|c|c|c|c|c|}
\hline Tumor markers & OM group & NOM group & $t$ & $p$ value \\
\hline Calcium (mmol/L) & $2.28 \pm 0.03$ & $2.25 \pm 0.01$ & 0.711 & 0.477 \\
\hline $\mathrm{HB}(\mathrm{g} / \mathrm{L})$ & $114.62 \pm 3.26$ & $119.08 \pm 0.56$ & -1.594 & 0.111 \\
\hline $\mathrm{ALP}(\mathrm{U} / \mathrm{L})$ & $117.40 \pm 8.74$ & $99.29 \pm 3.38$ & 1.099 & 0.272 \\
\hline $\mathrm{AFP}(\mathrm{ng} / \mathrm{mL})$ & $3.15 \pm 0.30$ & $1.86 \pm 0.04$ & 4.326 & $<0.001$ \\
\hline CEA (ng/mL) & $239.85 \pm 82.71$ & $62.67 \pm 9.02$ & 2.130 & 0.038 \\
\hline $\mathrm{NSE}(\mu \mathrm{g} / \mathrm{L})$ & $26.29 \pm 2.24$ & $23.90 \pm 1.06$ & 0.460 & 0.645 \\
\hline CYFRA21-1 (ng/mL) & $41.02 \pm 5.02$ & $9.81 \pm 1.05$ & 6.086 & $<0.001$ \\
\hline CA-125 (U/mL) & $413.05 \pm 84.25$ & $86.20 \pm 6.29$ & 3.869 & $<0.001$ \\
\hline CA-153 (U/mL) & $89.24 \pm 18.04$ & $22.83 \pm 1.08$ & 3.675 & 0.001 \\
\hline CA-199 (U/mL) & $158.59 \pm 50.57$ & $69.96 \pm 18.84$ & 0.963 & 0.336 \\
\hline TPSA (ng/L) & $4.28 \pm 0.33$ & $1.59 \pm 0.10$ & 7.759 & $<0.001$ \\
\hline
\end{tabular}

Independent sample $t$-test was applied. $p<0.05$ represented statistical significance. OM: ocular metastasis; NOM: nonocular metastasis.

TABLE 4: Risk factors of OM in patients with lung adenocarcinoma.

\begin{tabular}{lcccc}
\hline Factors & $\mathrm{B}$ & $\operatorname{Exp}(B)$ & OR $(95 \% \mathrm{CI})$ & $p$ value \\
\hline CEA & 0.000 & 1.000 & $0.999-1.000$ & 0.671 \\
CYFRA21-1 & -0.009 & 0.991 & $0.987-0.995$ & $<0.001$ \\
CA-125 & -0.001 & 0.999 & $0.998-1.000$ & 0.005 \\
CA-153 & -0.008 & 0.992 & $0.987-0.996$ & $<0.001$ \\
\hline
\end{tabular}

Binary logistic analysis was applied. $p<0.05$ represented statistical significance. $B$ : coefficient of regression; OR: odds ratio; CI: confidence interval; OM: ocular metastasis.

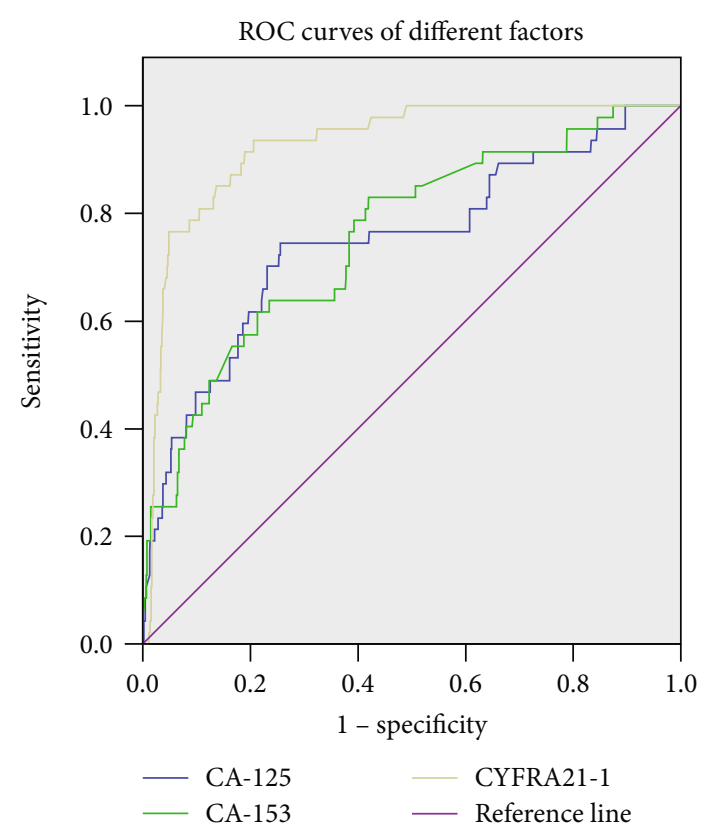

FIGURE 2: The ROC curves of risk factor for detecting OM in lung adenocarcinoma. ROC curves of CYFRA21-1, CA-123, and CA153 as a single risk factor of OM. ROC: receiver operating characteristic; OM: ocular metastasis.
CYFRA 21-1, CA-125, and CA-153 were identified as possible independent risk factors for OM in patients with lung adenocarcinoma. CYFRA 21-1 is a fragment of cytokeratin19 (an epithelial cell filament) produced during cancer cell differentiation. This marker has been associated with lung cancer, colorectal tumors, and bladder cancer [27-29], as well as with distant metastasis in patients with lung cancer [30]. In a retrospective study, Chen et al. [31] found that CYFRA 21-1 was an independent risk factor for predicting lymph node metastasis in lung cancer, with a sensitivity and specificity of 0.375 and 0.85 , respectively. CA-125, also known as tumor antigen 125 , is a high molecular weight glycoprotein produced by the MUC16 gene that is expressed on the surface of epithelial ovarian tumors and mesotheliumderived cells. CA-125 has been widely used to diagnose and assess progression and treatment effects for multiple types of cancer $[32,33]$ CA-125 levels were also found to be useful for predicting lymph node metastasis in patients with ovarian cancer [34]. CA-153 was first identified on breast cancer cell membranes with a relative molecular weight of 4000 . Abnormal CA-153 levels may also be measured in patients with many types of tumors including lung cancer [35]. It can be used to predict bone metastasis in breast cancer patients [36]. Based on the analyses of CYFRA 21-1, CA-125, and CA-153 levels and the high AUCs of the three biomarkers, we concluded that they are independent risk factors for OM in patients with lung adenocarcinoma.

The cutoff values showed that CYFRA 21-1>6.785 ng/ $\mathrm{mL}, \quad$ CA-125 $>66.295 \mathrm{U} / \mathrm{mL}$, and CA-125>13.005 U/mL were associated with $\mathrm{OM}$ in patients with lung adenocarcinoma. The highest AUC was observed for CYFRA 21-1, suggesting its superior diagnostic value in predicting OM. We also analyzed diagnostic accuracy rates for different combinations of these risk factors. However, the AUC values of these combinations were lower than that of CYFRA 21-1.

Some limitations of this study should be mentioned. First, the group of patients with OM was small, and these findings should be replicated in a larger cohort. Secondly, the participants were from the same hospital, which may limit the generalizability of our results. 
TABLE 5: The cutoff value, sensitivity, specificity, and AUC for single risk factor in predicting OM in lung adenocarcinoma patients.

\begin{tabular}{|c|c|c|c|c|c|}
\hline Factor & Cutoff value & Sensitivity (\%) & Specificity (\%) & AUC & $p$ \\
\hline CYFRA21-1 (ng/mL) & 6.785 & 0.936 & 0.794 & 0.928 & $<0.001$ \\
\hline CA-125 (U/mL) & 66.295 & 0.745 & 0.744 & 0.749 & $<0.001$ \\
\hline CA-153 (U/mL) & 13.005 & 0.830 & 0.580 & 0.758 & $<0.001$ \\
\hline CYFRA21-1+CA-125 & - & 0.814 & 0.851 & 0.890 & $<0.001$ \\
\hline CYFRA21-1+CA-153 & - & 0.769 & 0.872 & 0.880 & $<0.001$ \\
\hline CA-125+CA-153 & - & 0.682 & 0.809 & 0.809 & $<0.001$ \\
\hline CYFRA21-1+CA-125+CA-153 & - & 0.807 & 0.851 & 0.888 & $<0.001$ \\
\hline
\end{tabular}

Sensitivity and specificity were obtained at the point of cutoff value. $p<0.05$ represented statistical significance. AUC: area under the curve; CI: confidence interval; OM: ocular metastasis.

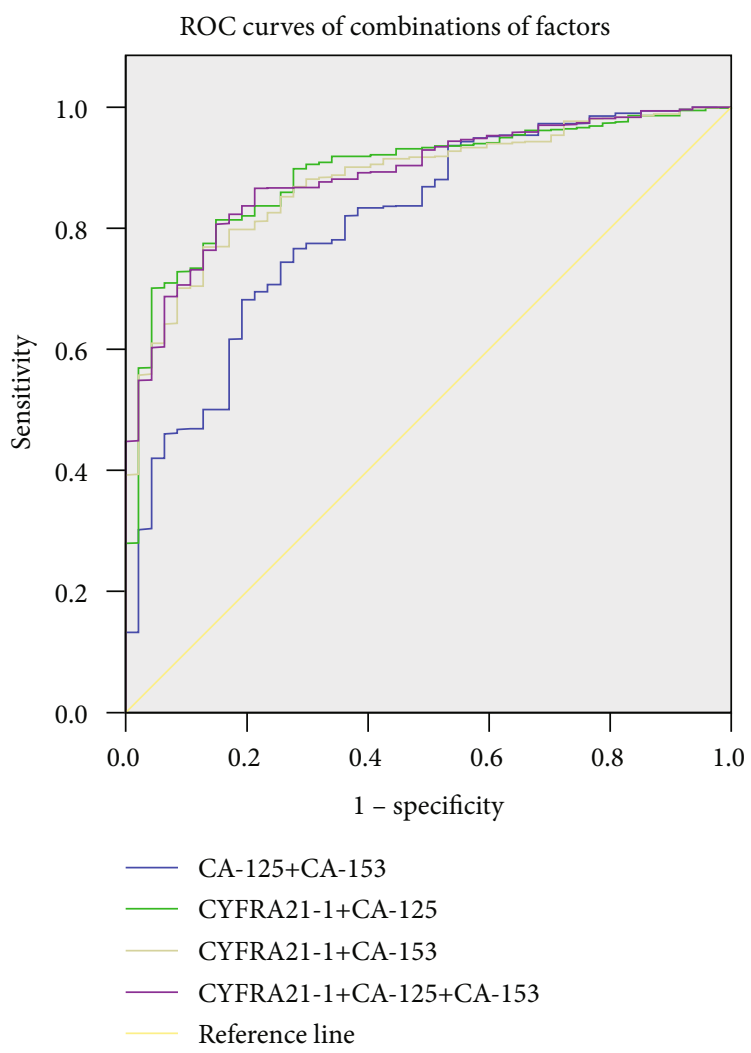

FIGURE 3: The ROC curves of combinations of different combinations of risk factors for detecting OM in lung adenocarcinoma patients and the ROC curves of CYFRA 21-1+CA-125, CYFRA 21-1+CA-153, CA-125+CA-153, and CYFRA 21-1+CA-125+CA-153. ROC: receiver operating characteristic; OM: ocular metastasis.

TABLE 6: The risk factors of metastases of lung cancer.

\begin{tabular}{llccc}
\hline Author & Year & Histopathological type & Metastatic sites & Risk factor \\
\hline Pollan [37] & 2003 & NSCLC & NS & CA-125 \\
Oshiro [38] & 2004 & Adenocarcinoma & Liver & AFP \\
Cabreraalarcon [39] & 2011 & NS & BS & CYFRA21-1 \\
Lee [30] & 2012 & NSCLC & Lymph node & CYFRA21-1, CEA \\
Chen [31] & 2015 & NS & Brain & NSE \\
Chen [40] & 2015 & NSCLC & Bone & CA-125, ALP \\
Zhou [41] & 2017 & NS & Intertrabecular vertebral & CEA \\
Morita [42] & 2019 & NSCLC & &
\end{tabular}

NS: not specific; NSCLC: non-small-cell lung cancer. 
Based on the analysis of 1153 patients with lung adenocarcinoma, we conclude that the serum concentrations of CYFRA 21-1, CA-125, and CA-153 are independent risk factors for OM. Among these, CYFRA 21-1 has the highest accuracy in predicting OM.

\section{Data Availability}

All data is available on request.

\section{Conflicts of Interest}

This was not an industry-supported study. The authors report no conflicts of interest in this work.

\section{Authors' Contributions}

Wen-Qing Shi and Wen-Feng Liu have contributed equally to this work.

\section{Acknowledgments}

This study was supported by the National Natural Science Foundation of China (Nos. 81660158, 81460092, and 81400372) and Natural Science Key Project of Jiangxi Province (No. 20161ACB21017).

\section{References}

[1] L. A. Torre, F. Bray, R. L. Siegel, J. Ferlay, J. Lortet-Tieulent, and A. Jemal, "Global cancer statistics, 2012," CA: a Cancer Journal for Clinicians, vol. 65, no. 2, pp. 87-108, 2013.

[2] M. Marel, F. Krejbich, P. Stránská et al., “Analysis of symptoms and case history data in the set of 353 patients with lung cancer," Casopís Lékar̆u Českých, vol. 148, no. 9, pp. 416-423, 2009.

[3] Y. Zhao, S. Wang, K. Aunan, H. M. Seip, and J. Hao, "Air pollution and lung cancer risks in China-a meta-analysis," Science of The Total Environment, vol. 366, no. 2-3, pp. 500-513, 2006.

[4] A. Fucic, M. Z. Gamulin, Z. Ferencic et al., "Lung cancer and environmental chemical exposure: a review of our current state of knowledge with reference to the role of hormones and hormone receptors as an increased risk factor for developing lung cancer in man," Toxicologic Pathology, vol. 38, no. 6, pp. 849-855, 2010.

[5] J. M. Tang, Q. Y. He, R. X. Guo, and X. J. Chang, "Phosphorylated Akt overexpression and loss of PTEN expression in non-small cell lung cancer confers poor prognosis," Lung Cancer, vol. 51, no. 2, pp. 181-191, 2006.

[6] Q. Z. Dong, W. Yang, X. J. Dong et al., "CIP2A is Overexpressed in Non-Small Cell Lungcancer and Correlates with Poor Prognosis," Annals of Surgical Oncology, vol. 18, no. 3, pp. 857-865, 2011.

[7] B. F. Fernandes, L. H. Fernandes, and M. N. Burnier Jr., "Choroidal mass as the presenting sign of small cell lung carcinoma," Canadian Journal of Ophthalmology, vol. 41, no. 5, pp. 605-608, 2006.

[8] B. George, W. J. Wirostko, T. B. Connor, and N. W. Choong, "Complete and durable response of choroid metastasis from non-small cell lung cancer with systemic bevacizumab and chemotherapy," Journal of Thoracic Oncology, vol. 4, no. 5, pp. 661-662, 2009.

[9] D. X. Nguyen, A. C. Chiang, X. H. Zhang et al., "WNT/TCF signaling through LEF1 and HOXB9 mediates lung adenocarcinoma metastasis," Cell, vol. 138, no. 1, pp. 51-62, 2009.

[10] C. D'Antonio, A. Passaro, B. Gori et al., "Bone and brain metastasis in lung cancer: recent advances in therapeutic strategies," Therapeutic Advances in Medical Oncology, vol. 6, no. 3, pp. 101-114, 2014.

[11] Z. Zhu and Y. Chai, "Complete remission of liver metastasis in a lung cancer patient with epidermal growth factor mutation achieved with Icotinib," Thorac Cancer, vol. 7, no. 6, pp. 681-683, 2016.

[12] I. Georgalas, T. Paraskevopoulos, C. Koutsandrea et al., "Ophthalmic Metastasis of Breast Cancer and Ocular Side Effects from Breast Cancer Treatment and Management: Mini Review," BioMed Research International, vol. 2015, Article ID 574086, 8 pages, 2015.

[13] C. L. Shields, S. Kaliki, G. S. Crabtree et al., "Iris metastasis from systemic cancer in 104 Patients," Cornea, vol. 34, no. 1, pp. 42-48, 2015.

[14] K. M. Kreusel, N. E. Bechrakis, T. Wiegel, L. Krause, and M. H. Foerster, "Incidence and clinical characteristics of symptomatic choroidal metastasis from lung cancer," Acta Ophthalmologica, vol. 86, no. 5, pp. 515-519, 2010.

[15] F. J. Ascaso, J. M. Castillo, F. J. García, J. A. Cristobal, A. Fuertes, and A. Artal, "Bilateral choroidal metastases revealing an advanced non-small cell lung cancer," The Annals of Thoracic Surgery, vol. 88, no. 3, pp. 1013-1015, 2009.

[16] L. Ma, X. W. Xie, H. Y. Wang, L. Y. Ma, and Z. G. Wen, "Clinical Evaluation of Tumor Markers for Diagnosis in Patients with Non-small Cell Lungcancer in China," Asian Pacific Journal of Cancer Prevention, vol. 16, no. 12, pp. 4891-4894, 2015.

[17] S. Cedrés, I. Nunez, M. Longo et al., "Serum Tumor Markers CEA, CYFRA21-1, and CA-125 Are Associated With Worse Prognosis In Advanced Non-Small-Cell Lung Cancer (NSCLC)," Clinical Lung Cancer, vol. 12, no. 3, pp. 172-179, 2011.

[18] P. C. Hoffman, A. M. Mauer, and E. E. Vokes, "Lung cancer," Lancet, vol. 355, no. 9202, pp. 479-485, 2000.

[19] S. U. Shah, A. Mashayekhi, C. L. Shields et al., "Uveal metastasis from lung cancer: clinical features, treatment, and outcome in 194 patients," Ophthalmology, vol. 121, no. 1, pp. 352-357, 2014.

[20] A. P. Ferry and R. L. Font, "Carcinoma metastatic to the eye and orbit. I. a clinicopathologic study of 227 cases," Archives of Ophthalmology, vol. 92, no. 4, pp. 276-286, 1974.

[21] R. Chew, J. Potter, and A. DiMattina, "Conjunctival metastasis as the presenting sign for stage IV lung cancer," Optometry and Vision Science, vol. 91, no. 2, pp. e38-e42, 2014.

[22] C. L. Shields, J. F. McMahon, H. T. Atalay, M. Hasanreisoglu, and J. A. Shields, "Retinal metastasis from systemic cancer in 8 cases," JAMA Ophthalmology, vol. 132, no. 11, pp. 1303-1308, 2014.

[23] S. Strohkamp, T. Gemoll, and J. K. Habermann, "Possibilities and limitations of 2DE-based analyses for identifying lowabundant tumor markers in human serum and plasma," Proteomics, vol. 16, no. 19, pp. 2519-2532, 2016.

[24] S. Kobayashi, A. Tomokuni, H. Takahashi et al., "The clinical significance of alpha-fetoprotein mRNAs in patients with 
hepatocellular carcinoma," Gastrointestinal Tumors, vol. 3, no. 3-4, pp. 141-152, 2017.

[25] H. Takeshita, S. Kawakami, A. Yano, Y. Okada, M. Morozumi, and T. YamadA, "Percent decrease of serum prostate-specific antigen after dutasteride administration is equivalent in men with clinical benign prostatic hyperplasia having baseline prostate-specific antigen $>10 \mathrm{ng} / \mathrm{mL}$ and those having baseline prostate-specific antigen 2," International Journal of Urology, vol. 24, no. 3, pp. 238-239, 2017.

[26] S. Chen, L. Wang, K. Qian et al., "Establishing a prediction model for prostate cancer bone metastasis," International Journal of Biological Sciences, vol. 15, no. 1, pp. 208-220, 2019.

[27] J. L. Pujol, J. M. Boher, J. Grenier, and X. Quantin, "Cyfra 21-1, neuron specific enolase and prognosis of non-small cell lung cancer: prospective study in 621 patients," Lung Cancer, vol. 31, no. 2-3, pp. 221-231, 2001.

[28] W. Zhong, Z. Yu, J. Zhan et al., "Association of serum levels of CEA, CA199, CA125, CYFRA21-1 and CA72-4 and disease characteristics in colorectal cancer," Pathology Oncology Research, vol. 21, no. 1, pp. 83-95, 2015.

[29] Y. Senga, G. Kimura, T. Hattori, and K. Yoshida, "Clinical evaluation of soluble cytokeratin 19 fragments (cyfra 21-1) in serum and urine of patients with bladder cancer," Urology, vol. 48, no. 5, pp. 703-710, 1996.

[30] D. S. Lee, Y. S. Kim, S. L. Jung et al., “The relevance of serum carcinoembryonic antigen as an indicator of brain metastasis detection in advanced non-small cell lung cancer," Tumour Biology, vol. 33, no. 4, pp. 1065-1073, 2012.

[31] F. Chen, C. E. Yan, J. Li, X. H. Han, H. Wang, and J. Qi, "Diagnostic value of CYFRA 21-1 and CEA for predicting lymph node metastasis in operable lung cancer," International Journal of Clinical and Experimental Medicine, vol. 8, no. 6, pp. 9820-9824, 2015.

[32] E. O. Hamed, H. Ahmed, O. B. Sedeek, A. M. Mohammed, A. A. Abd-alla, and H. M. Abdel Ghaffar, "Significance of HE4 estimation in comparison with CA125 in diagnosis of;ovarian cancer and assessment of treatment response," Diagnostic Pathology, vol. 8, no. 1, p. 11, 2013.

[33] Y. C. Zeng, R. Wu, S. L. Wang et al., "Serum CA125 level predicts prognosis in patients with multiple brain metastases from non-small cell lung cancer before and after treatment of whole-brain radiotherapy," Medical Oncology, vol. 31, no. 7, p. $48,2014$.

[34] S. Sudolmuş, N. Köroğlu, G. Yıldırım, V. Ulker, A. Gulkilik, and R. Dansuk, "Can CA-125 predict lymph node metastasis in epithelial ovarian cancers in Turkish population?," Disease Markers, vol. 2014, Article ID 492537, 6 pages, 2014.

[35] S. Sun, Z. Chen, C. Cao et al., "Diagnostic value of epidermal growth factor, cancer antigen 125 , and cancer antigen 15-3 in bronchoalveolar lavage fluid of lung cancer," Current Oncology, vol. 24, no. 3, pp. 180-184, 2017.

[36] W. Z. Chen, J. F. Shen, Y. Zhou, X. Y. Chen, J. M. Liu, and Z. L. Liu, "Clinical characteristics and risk factors for developing bone metastases in patients with breast cancer," Scientific Reports, vol. 7, no. 1, p. 11325, 2017.

[37] M. Pollan, G. Varela, A. Torres et al., "Clinical value of p53, cerbB-2, CEA and CA125 regarding relapse, metastasis and death in resectable non-small cell lung cancer," International Journal of Cancer, vol. 107, no. 5, pp. 781-790, 2003.

[38] Y. Oshiro, Y. Takada, T. Enomoto, K. Fukao, S. Ishikawa, and T. Iijima, "A resected case of metachronous liver metastasis from lung cancer producing alpha-fetoprotein (AFP) and protein induced by vitamin $\mathrm{K}$ absence or antagonist II (PIVKAII)," Hepato-Gastroenterology, vol. 51, no. 58, pp. 1144-1147, 2004.

[39] J. L. Cabreraalarcon, A. Carrillo-Vico, J. D. Santotoribio et al., "CYFRA 21-1 as a tool for distant metastasis detection in lung cancer," Clinical Laboratory, vol. 57, no. 11-12, pp. 1011-1014, 2011.

[40] Y. Chen, W. Peng, Y. Huang et al., "Significance of serum neuron-specific enolase before treatment in predicting brain metastases and prognosis of advanced non-small cell lung cancer," Zhonghua Zhong Liu Za Zhi, vol. 37, no. 7, pp. 508-511, 2015.

[41] Y. Zhou, Q. F. Yu, A. F. Peng, W. L. Tong, J. M. Liu, and Z. L. Liu, "The risk factors of bone metastases in patients with lung cancer," Scientific Reports, vol. 7, no. 1, p. 8970, 2017.

[42] S. Morita, T. Suda, C. Oda et al., "The value of $<\sup >18</$ sup $>$ F-FDG PET in the diagnosis of intertrabecular vertebral metastasis in a small cell lung cancer patient with a high serum CEA level," Internal Medicine, vol. 58, no. 3, pp. 415-418, 2019. 